\title{
Recent Advances in EELS Instrumentation and Analysis: Spectroscopy and Filtered Imaging with Extended Energy, Temporal, and Dynamic Range.
}

\author{
R.D. Twesten, M.M.G. Barfels, C.G. Trevor, P.J. Thomas, \\ N.K. Menon, A. Aitouchen and A.J. Gubbens \\ * Gatan Inc., 5794 W. Las Positas Blvd., Pleasanton, CA 94588, USA
}

The acquisition of high-quality EELS and energy-filtered image data in a transmission electron microscope (TEM) represents many challenges not experienced by most TEM acquisition modes. The central challenges are dose efficiency and dynamic range. For energy-filtered TEM (EFTEM), the data is acquired by broadly illuminating the sample while only acquiring a narrow band of energies; thus, the vast majority of the dose on the sample is wasted. Furthermore, as the energy range investigated is varied, the signal intensity changes dramatically, often over many orders of magnitude. For EELS, the challenges are even greater. The range of intensities of interest in a single spectrum can often span 6 to 7 orders of magnitude making recording problematic. Since the spectrum is recorded in parallel, EELS acquisition can be very dose efficient, but only if the acquisition device can be readout quickly and efficiently.

To address these issues, we have developed a next generation new post-column energy filter, the GIF Quantum ${ }^{\mathrm{TM}}$, which incorporates several new features that allow the optimal use of the highbrightness electron sources currently available. These improvements include:

$1 \mu$ s electrostatic shutter - An internal shutter system that allows exposure control down to $1 \mu$ s has obvious advantages for acquiring EELS and EFTEM data where the zero-loss signal may contain many times more electrons in $1 \mathrm{eV}$ than the entire rest of the spectrum spread over thousands of eV of energy loss (figure 1). The fast shutter is also pivotal in automating the acquisition process, thus reducing setup dose on the sample, and in enabling entirely new acquisition modes such as DualEELS ${ }^{\mathrm{TM}}$.

High-speed CCD Camera - By boosting the readouts rate of the CCD detector from 4 MPixels/s to $40 \mathrm{MPixels} / \mathrm{s}$, we have significantly reduced the time needed to acquire a frame of data, thus making the readout more dose efficient. For EELS acquisition, an even faster method of reading out the CCD was devised. Since each spectra only occupies a small fraction ( 260 lines) of the CCD, the camera only needs to clock the charge out of the way of the next incoming spectrum before opening the shutter. The data is stored or "stacked" on the CCD, and the readout rate and duty cycle of the detector is greatly improved. For a $1 \mathrm{kHz}$ spectral rate, the duty cycle of the detector is over $40 \%$. At $5 \mathrm{CCD}$ counts per primary electron, this readout configuration results in an electron counting rate of over $2.4 \times 10^{10}$ electrons/second over the entire 2048 energy channel spectrum.

DualEELS - The inherent intensity mismatch between the low-loss signal and the rest of the spectrum proves challenging for even the fastest camera and shutter. This was recognized early on by several groups[1,2]. However, the stacked readout and fast shutter technology can be used to project the low-loss spectrum on one part of the CCD and a higher energy loss spectrum on another part of the CCD. By properly sequencing the deflectors and a $<10 \mu$ s spectrum offset module, two independent spectra can be recorded with no mixing between the spectra and almost no additional 
readout overhead. By independently varying the exposure of the low- and core-loss data, the very intense zero-loss peak can be recorded along with the rest of the spectrum allowing full quantitative analysis of the spectrum (figure 2).

We will present details and advantages afforded by these new developments and show real world data collected under optimized conditions.

\section{References}

[1] J. Scott, P.J. Thomas, M. MacKenzie, S. McFadzean, J. Wilbrink, A.J. Craven, W.A.P. Nicholson Ultramicroscopy 108 (2008) 1586.

[2] H. Pinna, M. Tence, CNRS. (2006), Device for obtaining the image and/or spectra of electron energy loss FR2874124A1.
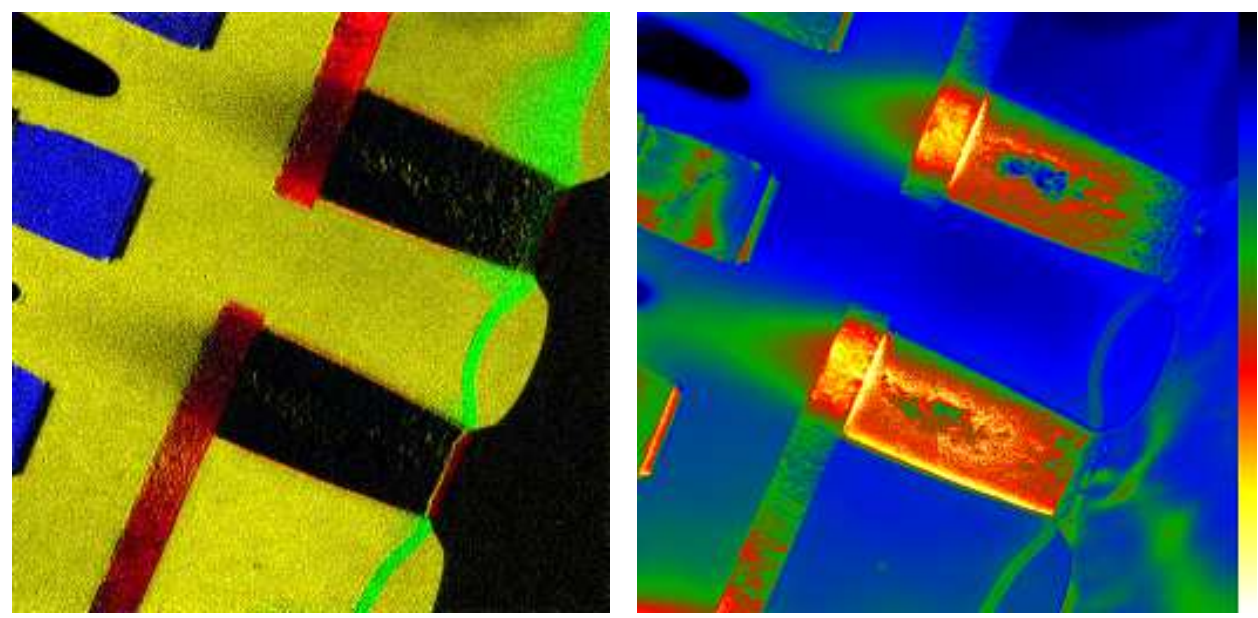

FIG. 1. The results of energy filtered mapping of a commercial semiconductor device. Left: Elemental color overlay map. Al-K (blue), O-K (yellow), Ti-L 23 (red), \& N-K (green); Right: Thickness map derived from zero-loss filtered / unfiltered image pair (black $=0$ white $=3.25 \mathrm{t} / \lambda$ ). Utilizing the fast shutter allowed the data to be recorded with a constant current density enabling direct comparisons of intensities spanning several orders of magnitude. Image width is $1.6 \mu \mathrm{m}$.

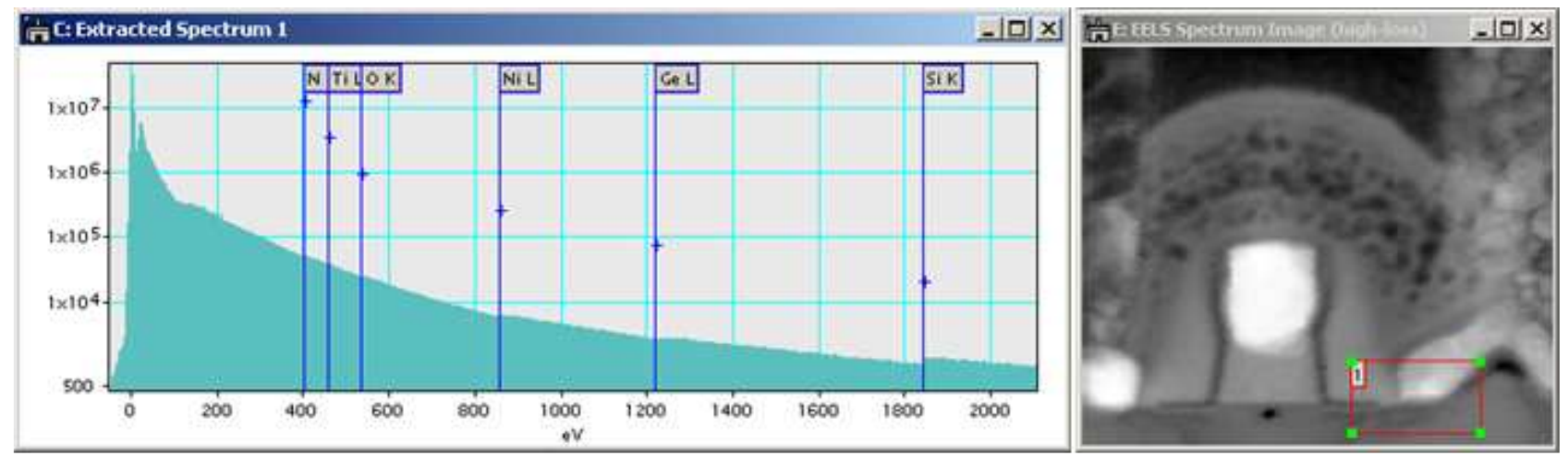

FIG. 2. Left: Spliced DualEELS data from the area indicated by "1" in the right figure. Right: Spectrum image data cube - For each pixel, both a low- and high-loss spectrum is acquired (integrated signal from $310-445 \mathrm{eV}$ is shown). Image width is $450 \mathrm{~nm}$. An ADF image (not shown) is capture during EELS data acquisition indicating precisely where the EELS data points are located. 